\title{
Erratum to: Identifying context and cause in small-N settings: a comparative multilevel analysis
}

\author{
Eva Thomann' ${ }^{1}$ Anita Manatschal ${ }^{2}$
}

Published online: 11 February 2016

(C) Springer Science+Business Media New York 2016

\section{Erratum to: Policy Sci \\ DOI 10.1007/s11077-015-9233-x}

In the original publication of the article, Table 1 has appeared incorrectly. The correct version of Table 1 is provided in this erratum.

The online version of the original article can be found under doi:10.1007/s11077-015-9233-X.

$\triangle$ Eva Thomann

eva.thomann@mzes.uni-mannheim.de

Anita Manatschal

anita.manatschal@ipw.unibe.ch

1 Mannheim Centre for European Social Research, University of Mannheim, 68131 Mannheim, Germany

2 Institute of Political Science, University of Bern, Fabrikstrasse 8, 3012 Bern, Switzerland 
Table 1 Research setting, policy instruments and refusal rates

\begin{tabular}{|c|c|c|c|c|c|c|c|}
\hline \multirow{2}{*}{$\begin{array}{l}\begin{array}{l}\text { Level of } \\
\text { analysis }\end{array} \\
\text { System II } \\
\text { (national) }\end{array}$} & \multirow{2}{*}{$\begin{array}{l}\text { Policy } \\
\text { instrument } \\
\text { Active public } \\
\text { awareness raising } \\
\text { Quality monitoring } \\
\text { Cost coverage } \\
\text { Presumed consent }\end{array}$} & \multicolumn{4}{|c|}{$\begin{array}{c}\text { CH } \\
\text { Context } 4\end{array}$} & \multicolumn{2}{|c|}{$\begin{array}{c}\text { ESP } \\
\text { Context } 5\end{array}$} \\
\hline & & $\begin{array}{l}\text { ar } \\
\text { qm } \\
\text { cc } \\
\text { pc }\end{array}$ & $\begin{array}{l}\text { ar } \\
\text { qm } \\
\text { cc } \\
\text { pc }\end{array}$ & $\begin{array}{l}\text { ar } \\
\mathrm{qm} \\
\mathrm{cc} \\
\mathrm{pc}\end{array}$ & $\begin{array}{l}\text { ar } \\
\text { qm } \\
\text { cc } \\
\text { pc }\end{array}$ & $\begin{array}{l}\text { AR } \\
\text { QM } \\
\text { CC } \\
\text { PC }\end{array}$ & $\begin{array}{l}\text { AR } \\
\text { QM } \\
\text { CC } \\
\text { PC }\end{array}$ \\
\hline $\begin{array}{l}\text { System I } \\
\text { (CH \& ESP } \\
\text { regions) }\end{array}$ & $\begin{array}{l}\text { Comprehensive } \\
\text { education of } \\
\text { hospital staff }\end{array}$ & \multicolumn{2}{|c|}{$\begin{array}{c}\text { CH-GE } \\
\text { Context } 1\end{array}$} & \multicolumn{2}{|c|}{$\begin{array}{c}\text { CH-L } \\
\text { Context } 2\end{array}$} & \multicolumn{2}{|c|}{$\begin{array}{l}\text { ESP } \\
\text { Context } 3\end{array}$} \\
\hline \multirow[t]{2}{*}{$\begin{array}{l}\text { Subsystem } \\
\text { (cases) }\end{array}$} & $\begin{array}{l}\text { Active family } \\
\text { approach } \\
\text { Donor coordinator } \\
\text { reimbursement } \\
\text { Donor transfer for } \\
\text { retrieval }\end{array}$ & $\begin{array}{c}\text { Large } \\
\text { hospitals } \\
\text { fam } \\
\text { RC } \\
t\end{array}$ & $\begin{array}{c}\text { Small } \\
\text { hospitals } \\
\text { fam } \\
\text { rc } \\
\text { T }\end{array}$ & $\begin{array}{c}\text { Large } \\
\text { hospitals } \\
\text { fam } \\
\text { RC } \\
t\end{array}$ & $\begin{array}{c}\text { Small } \\
\text { hospitals } \\
\text { fam } \\
\text { RC } \\
\text { T }\end{array}$ & $\begin{array}{c}\text { Large } \\
\text { hospitals } \\
\text { FAM } \\
\text { RC } \\
\text { t }\end{array}$ & $\begin{array}{c}\begin{array}{c}\text { Small } \\
\text { hospitals }\end{array} \\
\text { FAM } \\
\text { RC } \\
t\end{array}$ \\
\hline & Refusal rate & $49.0 \%$ & $78.0 \%$ & $28.4 \%$ & $75.0 \%$ & $19.8 \%$ & $16.2 \%$ \\
\hline
\end{tabular}

Capital letters $=$ policy instrument is applied. Lower case letters = policy instrument is not applied CH: Switzerland, CH-GE: German part of Switzerland, CH-L: Latin (i.e. French and Italian) part of Switzerland, ESP: Spain. Refusal rate = number of refusals by next of kin as share of total requests (in \%) Data sources: Swiss Donor Action (mean of 2007, 2008, 2009), Organicazión Nacional de Trasplantes (ONT 2009) 